\title{
Exploration of the Mechanism of Lianhua Qingwen in Treating Influenza Virus Pneumonia and New Coronavirus Pneumonia with the Concept of "Different Diseases with the Same Treatment" Based on Network Pharmacology
}

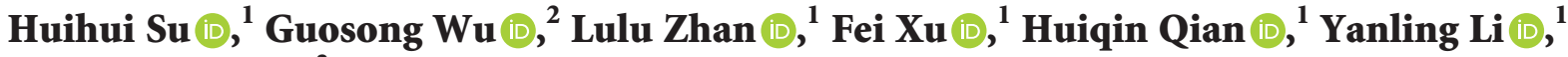 \\ and Ximei Zhu ${ }^{3}{ }^{3}$ \\ ${ }^{1}$ College of Pharmacy, Sanquan College of Xinxiang Medical University, Xinxiang 453000, China \\ ${ }^{2}$ Department of Pharmacy, Baiyun Branch of Nanfang Hospital of Southern Medical University, Guangzhou 510599, China \\ ${ }^{3}$ Clinical Pharmacists, The Maternal and Child Health Care Hospital of HuaDu District (Huzhong Hospital), \\ Guangzhou 510800, China
}

Correspondence should be addressed to Huihui Su; 1005071498@qq.com and Ximei Zhu; 719178189@qq.com

Received 21 January 2021; Revised 24 November 2021; Accepted 7 January 2022; Published 8 February 2022

Academic Editor: Sergio R. Ambrosio

Copyright (c) 2022 Huihui Su et al. This is an open access article distributed under the Creative Commons Attribution License, which permits unrestricted use, distribution, and reproduction in any medium, provided the original work is properly cited.

The 31 main components of Lianhua Qingwen (LHQW) were obtained through a literature and database search; the components included glycyrrhizic acid, emodin, chlorogenic acid, isophoroside A, forsythia, menthol, luteolin, quercetin, and rutin. Sixtyeight common targets for the treatment of novel coronavirus pneumonia (NCP) and influenza virus pneumonia (IVP) were also obtained. A "component-target-disease" network was constructed with Cytoscape 3.2.1 software, and 20 key targets, such as cyclooxygenase2 (COX2), interleukin-6 (IL-6), mitogen-activated protein kinase14 (Mapk14), and tumor necrosis factor (TNF), were screened from the network. The David database was used to perform a Kyoto Encyclopedia of Genes and Genomes (KEGG) signal pathway enrichment analysis and gene ontology (GO) biological process enrichment. Results showed that the key targets of LHQW in the treatment of NCP and IVP mainly involved biological processes, such as immune system process intervention, cell proliferation, apoptosis and invasion, toxic metabolism, cytokine activity, and regulation of the synthesis process. KEGG enrichment analysis revealed that 115 signalling pathways were related to the treatment of LHQW. Amongst them, IL-17, T cell receptor, Th17 cell differentiation, TNF, toll-like receptor, MAPK, apoptosis, and seven other signalling pathways were closely related to the occurrence and development of NCP and IVP. Molecular docking showed that each component had different degrees of binding with six targets, namely, 3C-like protease (3CL), angiotensin-converting enzyme 2 (ACE2), COX2, hemagglutinin (HA), IL-6, and neuraminidase (NA). Rutin, isoforsythiaside A, hesperidin and isochlorogenic acid B were the best components for docking with the six core targets. The first five components with the best docking results were isoforsythiaside, hesperidin, isochlorogenic acid B, forsythin E, and quercetin. In conclusion, LHQW has many components, targets, and pathways. The findings of this work can provide an important theoretical basis for determining the mechanism of LHQW in treating NCP and IVP.

\section{Background}

Lianhua Qingwen (LHQW) is composed of 13 traditional Chinese medicines (TCMs), namely, Forsythiae Fructus (Lianqiao, LQ), Lonicerae Japonicae Flos (Jinyinhua, JYH), Ephedare Herba (Mahuang, MH), Armeniacae Semen Amarum (Kuxingren, KXR), Gypsum Fibrosum (Shigao, SG),
Isatdis Radix (Banlangen, BLG), Dryopteridis Crassirhizomatis Rhizoma (Mianmaguanzhong, MMGZ), Houttuyniae Herba (Yuxingcao, YXC), Pogostemonis Herba (Guanghuoxiang, GHX), Rhei Radix et Rhizoma (Dahuang, DH), Rhodiolae Crenulatae Radix et Rhizoma (Hongjingtian, HJT), l-Menthol (Bohenao, BHN), and Glycyrrhizae Radix et Rhizoma (Gancao, GC). According to the pathological theory of TCM, LHQW is 
good medicine for dredging collaterals, dispersing the lung, opening with pungent and bitter drugs, and clearing away heat and detoxification [1]. It exerts good effects on influenza virus pneumonia (IVP) and has antibacterial, antipyretic, anti-inflammatory, antitussive, expectorant, and immune-regulating functions [2]. LHQW has no difference with oseltamivir [3] (oseltamivir is a neuraminidase inhibitor used in the treatment of influenza A and B) in terms of anti-influenza capability, but it is superior to oseltamivir in relieving the symptoms of influenza, especially fever, cough, headache, muscle soreness, and fatigue. It can also block the vicious cycle of multiple pathological links and plays a role in overall regulation and multitarget treatment. In addition, LHQW, as an effective drug for novel coronavirus pneumonia (NCP) diagnosis and treatment (Trial Seventh Edition issued by China in 2019), exerts a significant clinical effect on the treatment of coronavirus disease 2019 (COVID-19). Multiple retrospective analyses have shown that LHQW can significantly improve the clinical symptoms of fever, cough, expectoration, shortness of breath, and other common symptoms experienced by patients diagnosed with COVID-19; it can also alleviate the disease's condition and shorten its course [4-6]. LHQW has a significant effect on the treatment of IVP and NCP, but no systematic study has been conducted on the molecular mechanism of LHQW treatment of the two diseases.

Given the complexity of the composition and mechanism of traditional Chinese medicine (TCM), systematically explaining the mechanism of TCM in the treatment of diseases is difficult. Network pharmacology is a new technology that integrates system biology, multidirectional pharmacology, and computer analysis. It can systematically link drugs with diseases and observe the intervention and influence of drugs on disease networks [7-9]. The systematicness of network pharmacology coincides with the integrity of TCM in treating diseases.

In recent years, network pharmacology has been widely used to systematically explain the mechanism of action of TCM and TCM compound prescription in the treatment of diseases [10-12], and extensive research has been conducted on the "different diseases with the same treatment" concept of TCM [13]. Examples include the mechanism of brain and heart treatment of Danhong injection [14]; the mechanism of network pharmacology of Jiaotai pills for diabetes, depression, and insomnia [15]; the mechanism of network pharmacology of Shengmai Yin in treating diabetes and heart failure [16]; and analysis of Chaihu Guizhi decoction based on the network pharmacology model for the treatment of gastric ulcer and epilepsy [17]. In this work, we aim to use a comprehensive network pharmacology-based approach to investigate the mechanism of how LHQW exerts therapeutic effects on viral pneumonia.

\section{Materials and Methods}

2.1. Retrieval of the Main Components of LHQW and Prediction of Its Target. The main components of LHQW can be identified and quantified through literature retrieval. Through verification and conversion of the PubChem database (https://pubchem.ncbi.nlm.nih.gov/), the target protein of the compound was obtained from the Traditional Chinese Medicine Systems Pharmacology (TCMSP) database (http://tcmspw.com/tcmsp.php), National Center for Biotechnology Information (NCBI) database (https:// pubchem.ncbi.nlm.nih.gov/), and https://dx.doi.org/10. 1093/nar/gkz382 (SWISS) database (http://www. swisstargetprediction.ch/). At the same time, the protein database UniProt (http://www.uniprot.org/uploadlists/) was used to convert the targets to a unified gene name.

2.2. Identification of Antiviral Pneumonia-Related Targets of LHQW. GeneCards database (https://www.genecards.org/) is a platform that provides all known human genes in genome, proteome, transcription, heredity, and function [18]. NCP- and IVP-related target information were collected by searching for the keywords "influenza virus pneumonia" and "novel coronavirus pneumonia." The IVP-associated targets, NCP-related targets, and compound targets were mapped in Venny 2.1.0 software, and the common targets were screened out and regarded as a potential target for LHQW to fight viral pneumonia.

\subsection{Construction of "Native Component-Target" Network and} Screening of Key Targets. To determine the interaction between viral pneumonia potential therapeutic targets and targets of LHQW, this study introduced the selected targets into the STRING network platform (https://string$\mathrm{db} . \mathrm{org} /$ ) to construct a protein-protein interaction (PPI, the process of combining two or more proteins to determine their biochemical functions) network map. The database parameter was set to "Homo sapiens," and the protein type was set to the rating criterion (confidence level >0.9). Then, the PPI network file was downloaded. The active components, their corresponding LHQW targets, and PPI were input into Cytoscape 3.2.1 to construct the "native component-target" network of LHQW. The topology parameters of the network were analysed using the network analyser function of Cytoscape and derived. The related topological data, such as degree (DG) and betweenness centrality (BC), were examined. DG and $\mathrm{BC}$ are important topological parameters for evaluating the specific gravity of a node in the network. DG can reflect the number of links between one node and other nodes in the network, and $\mathrm{BC}$ reflects the ratio of the number of paths passing through the node to the total number of shortest paths in all the shortest paths in the network. The larger the topology parameters of the node are, the more critical the target in the network is $[19,20]$.

2.4. KEGG Signal Pathway and GO Biological Process Enrichment Analyses. The core targets of LHQW in the treatment of viral pneumonia were subjected to gene ontology (GO) biological process and Kyoto Encyclopedia of Genes and Genomes (KEGG) signal pathway enrichment analyses by using the David database (https://david.ncifcrf. gov/). GO enrichment can be used to understand the main 
action process of the target. The KEGG pathway can be adopted to understand the role of this target in metabolism, signal transduction and other pathways by observing the distribution of targets in the pathway. The main signalling pathways and biological processes involved in the efficacy of LHQW were obtained by screening the target genes with $P<0.05$. The $P$ value is the statistical significance of target enrichment/annotation, and $P<0.05$ indicates that the pathway has analytical significance. The smaller the $P$ value is, the more significant the enrichment result is.

2.5. Molecular Docking and Data Processing. The protein structure file was downloaded from the Protein Data Bank (PDB) website (https://www.rcsb.org/), and the target protein and component were pretreated using AutoDock 1.5.6 and Discovery Studio 2.5 software. Then, the target and component were docked using AutoDock Vina. Generally, negative values of binding energy are associated with the stable conformation of ligand binding to the receptor. If the binding energy is less than $-5 \mathrm{kcal} / \mathrm{mol}$, then the ligand and receptor can bind spontaneously; binding energy $\leq-7 \mathrm{kcal} /$ mol denotes good binding [21, 22]. The flowchart of the experimental procedures of our study is shown in Figure 1.

\section{Results}

3.1. Main Components and Corresponding Targets of LHQW. After searching previous studies [23-26], we obtained 31 active components that could be detected qualitatively and quantitatively in LHQW. The composition analysis of LHQW shown above indicated that Lonicerae Japonicae Flos had 10 chemical constituents, Forsythiae Fructus had 9, Pogostemon Cablin (Blanco) Benth had 6, and Isatdis Radix had 4. Ephedra Herba, Houttuyniae Herba, Radix Rhei et Rhizome, and Licorice each had 3 chemical constituents. Menthae Herba had 2 chemicals, and Rhodiola rosea L. and Amygdalus Communis Vas each had 1 chemical constituent (Table 1).

In this study, 104, 414, and 341 predicted targets were obtained from TCMSP, NCBI, and SWISS databases, respectively. After UniProt correction and elimination of duplicate items, a total of 712 targets related to LHQW were obtained (Table 2).

3.2. Identification of Potential Targets of LHQW in Fighting Viral Pneumonia. The NCP- and IVP-related targets were derived from the GeneCards database by using "novel coronavirus pneumonia" and "influenza virus pneumonia" as the keywords, respectively. The NCP- and IVP-related targets from the GeneCards database numbered 259 and 1326, respectively. These targets were mapped in Venny 2.1.0 to map the 68 common targets (Figure 2). These targets are common targets related to the development of NCP and IVP, and they are the reason why LHQW can be used to treat NCP and IVP. The other NCP-related targets are 3C-like protease (3CL) and angiotensin-converting enzyme 2 (ACE2). The most representative IVP targets are neuraminidase (NA) and hemagglutinin (HA). They embody the characteristics of seeking common ground whilst reserving differences in the process of "plague," as indicated in TCM.

Potential target of LHQW intervention for viral pneumonia (yellow circle: the larger the topology parameter is, the larger the target circle is). Main ingredients of LHQW (red triangle) and medicinal composition of LHQW (green diamond).

3.3. Construction and Analysis of the "Native ComponentTarget" Network. To further explore the mechanism of LHQW, 68 target proteins of LHQW against viral pneumonia were input into the STRING database, and the PPI network file was obtained (Figure 3). The medicinal materials, components, and PPI network were imported into Cytoscape software to construct the "herb-component-target" network of LHQW against viral pneumonia (Figure 4). The network has 109 nodes (including 31 compound nodes, 68 target protein nodes, and 11 kinds of medicinal materials) and 222 edges. Each candidate compound acts on an average of 17.4 targets, and the average number of drugs linked to each target protein is 6.6, indicating that LHQW has the characteristics of multicomponent and multitarget in the treatment of diseases. In combination with the analysis of network topology, the DG and $\mathrm{BC}$ of a network node are common indicators to describe the importance of the node. The median of DG in the targets is 20 , and the median of BC in the targets is 0.003 . A total of 20 nodes are more than twice the value and twice the center of this value (Table 3), suggesting that these targets are also the key factors to LHQW's treatment of viral pneumonia.

3.4. GO Biological Process Analysis. Twenty key targets were annotated with GO terms, and 91 terms with $P<0.05$ were screened out. In general, the GO terms were classified into three parts: biological process (BP), molecular function (MF), and cellular component (CC). The visualisation results of BP, CC, and MF enrichment analyses were obtained using OmicShare, as shown in Figure 5. These targets are mainly distributed in the extracellular matrix, intercellular synapse, membrane sealing cavity, organelles, membrane system, and other cellular components. They play roles in molecular functions, such as molecular function regulation, binding, antioxidant activity, catalytic activity, and transcriptional regulation activity and participate in immune system intervention, cell proliferation, cell apoptosis, cell invasion, toxicant metabolism cytokine activity, and synthesis process regulation. These results suggest that LHQW functions against viral pneumonia by intervening in the above-mentioned biological processes.

3.5. KEGG Signal Pathway Enrichment Analysis. The enrichment analysis of KEGG signalling pathways was carried out on 20 key targets, and 115 signal pathways with $P<0.05$ were selected. The visualisation results of the top 20 KEGG pathways with the most significant $P$ value were obtained using OmicShare, as shown in Figure 6. These pathways are mainly involved in immunity (T cell receptor/IL-17/Th17 


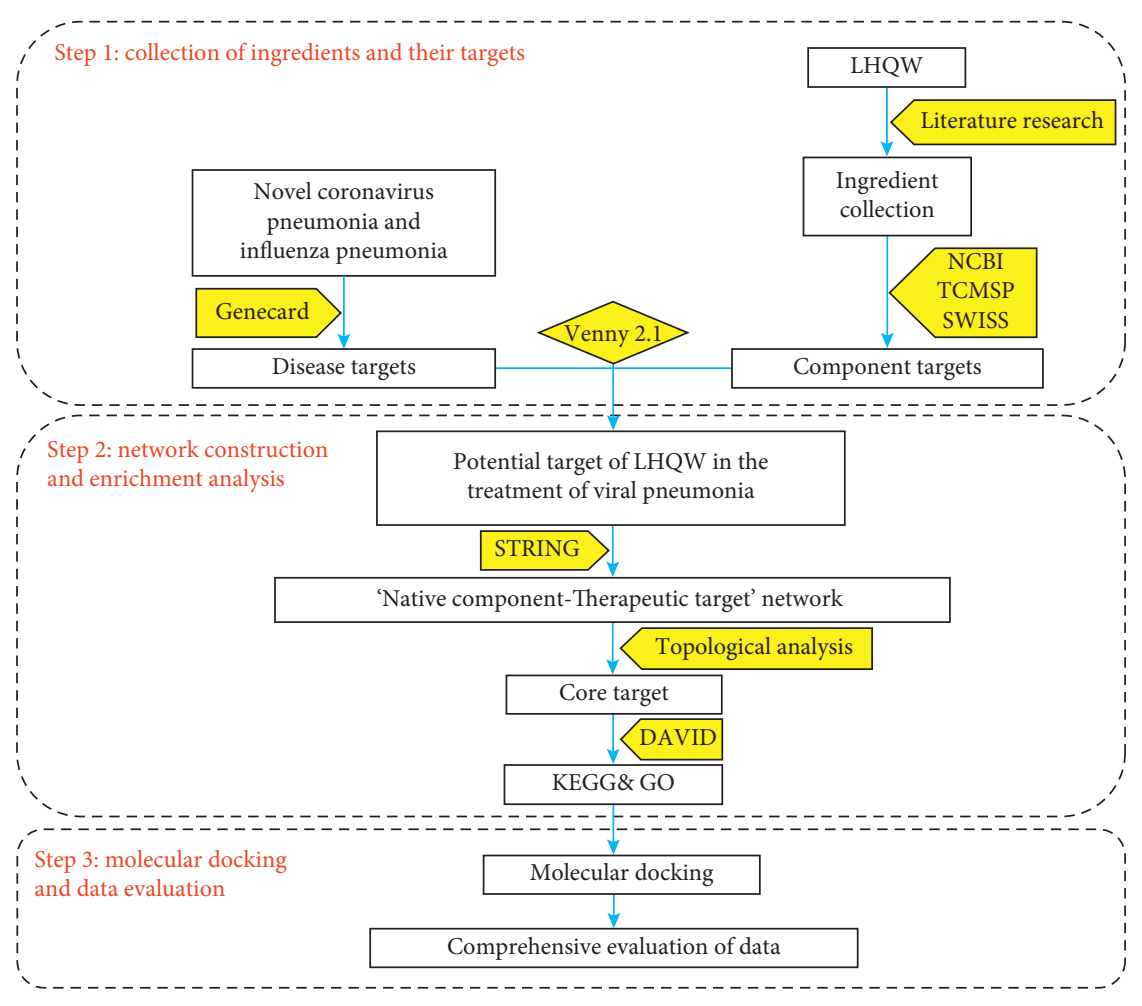

FIgURE 1: Flowchart of the research.

cell differentiation signalling pathway), inflammation (TNF/ toll-like receptor (TLR) signalling pathway), signal transduction (MAPK signalling pathway, etc.), cell apoptosis (apoptosis signalling pathway), and other pathways involved in influenza $\mathrm{A}, \mathrm{B}$, and $\mathrm{C}$ viral hepatitis. These results suggest that LHQW may play an integral role in the treatment of viral pneumonia by intervening in many pathways related to immunity, inflammation, viruses, and so on.

3.6. Molecular Docking. The occurrence and development of a new coronavirus and influenza virus exhibit similarities and differences after infection. The former mainly involves two targets (3CL and ACE2) [27-30], whereas the latter is mainly related to NA and HA [31-33]. Although the initial routes of infection are different, both cause a cytokine storm and further worsen the patient's condition [34, 35]. According to the results of this study, COX2 and IL-6 are involved in the infection. Therefore, this study further explored the material basis of LHQW in the treatment of viral pneumonia. The docking results showed that each component had different degrees of binding to each target (Figure 7), and the binding energies with 3CL, ACE2, NA, HA, COX2, and IL- 6 were lower than $-7.0 \mathrm{kcal} / \mathrm{mol}$.

The top five components for combination with each target are shown in Figure 8. Amongst them, rutin, isoforsythiaside $\mathrm{A}$, hesperidin, and isochlorogenic acid $\mathrm{B}$ were the best components to combine with the six targets. COX2 and IL-6 were the most important common targets of LHQW against viral pneumonia, and the material basis of intervention was the same. However, differences were observed in the key targets of IVP (HA, NA) and NCP (3CL, ACE2) infection, and the material basis of intervention was also emphasised. The main components of anti-IVP are rutin, isoforsythiaside A, and hesperidin, and the main components of anti-NCP were isochlorogenic acid B and forsythin E.

To further analyse the results of each component combined with the targets, this study performed a multi-index comprehensive analysis and multi-index data processing by referring to the general evaluation normalisation method reported in literature [36, 37]. In accordance with Hassan's method (mathematical statistical method that can synthesise various indexes and express the final effect on $\mathrm{OD}$ ), the docking data of each component and six indicators, namely, 3CL, ACE2, COX2, HA, IL-6, and NA, were processed to calculate the geometric mean of the normalised values of each index, that is, the overall desirability (OD). $D_{\text {imax }}=\left(Y_{i}-Y_{\min }\right) /$ $\left(Y_{\max }-Y_{\min }\right)$, and $\mathrm{OD}=\left(D_{1} D_{2} \ldots \mathrm{DK}\right)^{1 / k}(k$ is the index number) $[38,39]$. The results showed that the first five components of the comprehensive index of 3CL, ACE2, COX2, HA, IL-6, and NA from large to small were isoforsythiaside A, hesperidin, isochlorogenic acid $\mathrm{B}$, forsythin $\mathrm{E}$, and quercitrin. The binding energies of isochlorogenic acid $\mathrm{B}$ with the six targets, namely, 3CL, ACE2, COX2, HA, IL-6, and NA, were all less than $-7 \mathrm{kcal} / \mathrm{mol}$, suggesting that these components play a key role in the treatment of viral pneumonia with LHQW. The results are shown in Table 4.

\section{Discussion}

In this study, 31 main components of LHQW were qualitatively and quantitatively obtained by compiling literature, and 712 component targets were obtained from three 
TABLE 1: Effective chemical constituents of LHQW and ownership of the medicinal materials.

\begin{tabular}{|c|c|c|}
\hline Number & English name & $\begin{array}{c}\text { Attribution of medicinal } \\
\text { materials }\end{array}$ \\
\hline 1 & Emodin & $\mathrm{DH}$ and $\mathrm{BLG}$ \\
\hline 2 & Rhein & $\mathrm{DH}$ and $\mathrm{BLG}$ \\
\hline 3 & Glycyrrhizic acid & GC and BLG \\
\hline 4 & Liquiritin & GC and BLG \\
\hline 5 & Chrysophanol & BLG \\
\hline 6 & Chlorogenic acid & JYH and LQ \\
\hline 7 & 4-Caffeoylquinic acid & JYH and YXC \\
\hline 8 & Isochlorogenic acid B & JYH \\
\hline 9 & $\begin{array}{l}\text { Isochlorogenic acid } \\
\text { C }\end{array}$ & JYH \\
\hline 10 & Isoforsythiaside & LQ \\
\hline 11 & Phillyrin & LQ \\
\hline 12 & Forsythoside E & LQ \\
\hline 13 & Rutin & $\begin{array}{c}\text { GC, BLG, JYH, LQ, MH, and } \\
\text { YXC }\end{array}$ \\
\hline 14 & Salidroside & HJT \\
\hline 15 & (-)-Phillygenin & LQ \\
\hline 16 & Mollugin & JYH \\
\hline 17 & Amygdalin & KXR \\
\hline 18 & L-Menthol & BHN \\
\hline 19 & Patchouli alcohol & GHX \\
\hline 20 & Guaiene & GHX \\
\hline 21 & $\alpha$-Gurjunene & $\mathrm{BHN}$ and $\mathrm{MH}$ \\
\hline 22 & $\alpha$-Bulnesene & GHX \\
\hline 23 & $\beta$-Patchoulene & GHX \\
\hline 24 & $\alpha$-Patchoulene & GHX \\
\hline 25 & Quercitrin & YXC \\
\hline 26 & Aloe emodin & $\mathrm{DH}$ \\
\hline 27 & Luteolin & $\mathrm{JYH}, \mathrm{LQ}$, and $\mathrm{MH}$ \\
\hline 28 & Hesperidin & $\mathrm{MH}$ \\
\hline 29 & Hyperin & GHX, LQ, JYH, and YXC \\
\hline 30 & Caffeic acid & JYH and LQ \\
\hline 31 & Neochlorogenic acid & JYH and YXC \\
\hline
\end{tabular}

Note. The acronyms in the column "Attribution of medicinal materials" are shown in the first paragraph of "Background."

databases. A total of 68 common targets for the treatment of viral pneumonia were obtained. The expression of COX2 is abnormal in IVP and NCP [40-43] and mainly distributed in endothelial cells, macrophages, lymphocytes, and so on under inflammatory conditions. The expression of COX2 in lung tissue increases exponentially, and the induced PEG2 accumulates in the lesions, causing the body to produce symptoms, such as redness, swelling, and pain. It also induces a cascade reaction in a vicious cycle, promotes the synthesis of other cytokines, including inflammatory factors (IL-6, TNF- $\alpha$, IL1, etc.), and chemokines (CXCL10, IL-8, etc.), and upregulates the expression of COX2 and NOS2, which induce the synthesis of inflammatory mediators, such as PGE2 and NO $[44,45]$. These series of reactions aggravate the inflammatory injury of lung tissue and worsen the patient's condition continuously. In addition, overexpression of COX2 can promote cell proliferation, inhibit apoptosis, inhibit the immune response, and evade immune monitoring [46, 47], such as inhibition of mitochondrial cytochrome C leakage and CASP3/9 activation. This biological process can break the balance between cell proliferation and
TABLE 2: Predicted targets for potential active components of LHQW.

\begin{tabular}{|c|c|c|c|c|c|}
\hline \multirow{2}{*}{$\begin{array}{l}\text { Serial } \\
\text { number }\end{array}$} & \multirow{2}{*}{ Ingredient } & \multicolumn{3}{|c|}{ Database } & \multirow{2}{*}{$\begin{array}{c}\text { Target } \\
\text { Total }\end{array}$} \\
\hline & & TCMSP & NCBI & SWISS & \\
\hline 1 & Emodin & 35 & 78 & 38 & 151 \\
\hline 2 & Rhein & 30 & 5 & 82 & 117 \\
\hline 3 & Glycyrrhizic acid & 0 & 69 & 29 & 98 \\
\hline 4 & Liquiritin & 22 & 18 & 20 & 60 \\
\hline 5 & Chrysophanol & 30 & 5 & 28 & 63 \\
\hline 6 & Chlorogenic acid & 7 & 67 & 39 & 113 \\
\hline 7 & $\begin{array}{l}\text { 4-Caffeoylquinic } \\
\text { acid }\end{array}$ & 7 & 7 & 39 & 53 \\
\hline 8 & $\begin{array}{l}\text { Isochlorogenic } \\
\text { acid B }\end{array}$ & 2 & 13 & 44 & 59 \\
\hline 9 & $\begin{array}{l}\text { Isochlorogenic } \\
\text { acid C }\end{array}$ & 3 & 15 & 46 & 65 \\
\hline 10 & Isoforsythiaside & 0 & 0 & 14 & 14 \\
\hline 11 & Phillyrin & 1 & 37 & 7 & 45 \\
\hline 12 & Forsythoside E & 2 & 0 & 21 & 23 \\
\hline 13 & Rutin & 13 & 71 & 21 & 105 \\
\hline 14 & Salidroside & 0 & 79 & 23 & 102 \\
\hline 15 & (-)-Phillygenin & 27 & 30 & 28 & 85 \\
\hline 16 & Mollugin & 21 & 41 & 86 & 148 \\
\hline 17 & Amygdalin & 19 & 59 & 32 & 110 \\
\hline 18 & L-Menthol & 11 & 28 & 32 & 71 \\
\hline 19 & Patchouli alcohol & 0 & 0 & 9 & 9 \\
\hline 20 & Guaiene & 26 & 8 & 3 & 37 \\
\hline 21 & $\alpha$-Gurjunene & 12 & 3 & 5 & 20 \\
\hline 22 & $\alpha$-Bulnesene & 0 & 15 & 0 & 15 \\
\hline 23 & $\beta$-Patchoulene & 11 & 26 & 10 & 47 \\
\hline 24 & $\alpha$-Patchoulene & 7 & 0 & 11 & 18 \\
\hline 25 & Quercitrin & 8 & 69 & 22 & 99 \\
\hline 26 & Aloe emodin & 24 & 78 & 6 & 108 \\
\hline 27 & Luteolin & 57 & 77 & 46 & 180 \\
\hline 28 & Hesperidin & 6 & 78 & 28 & 112 \\
\hline 29 & Hyperin & 10 & 27 & 30 & 67 \\
\hline 30 & Caffeic acid & 10 & 62 & 64 & 136 \\
\hline 31 & $\begin{array}{l}\text { Neochlorogenic } \\
\text { acid }\end{array}$ & 1 & 41 & 39 & 81 \\
\hline Total & mber of targets & 402 & 1106 & 1002 & 2411 \\
\hline Total & mber of targets & 104 & 414 & 341 & 712 \\
\hline
\end{tabular}

cell death, creating favourable conditions for pathogen breeding and allowing pathogens to take advantage of it. IL is one of the key common targets, and it includes IL-2, IL-6, and IL-10. IL- 6 is produced by monocyte macrophages, Th2 cells, and vascular endothelial cells $[48,49]$. NOS2 can promote the synthesis of IL-6 and participate in the inflammatory reaction by producing nitric oxide. MAPK1/3/8/ 14 and NF- $\kappa \mathrm{B}$, as regulatory factors of the inflammatory response, bind to the IL- 6 response element in the promoter of the acute phase protein gene $[50,51]$. When the body is activated by cytokines, neurotransmitters, hormones, cell stress, and cell adhesion, they jointly regulate various important physiological and pathological processes, such as inflammatory response, cell growth, differentiation, and stress adaptation. TNF- $\alpha$ is an important member of inflammatory cytokines $[52,53]$ that is mainly composed of activated macrophages, NK cells, and T cells. TNF- $\alpha$ can regulate immune cells, and as an endogenous heat source, it 


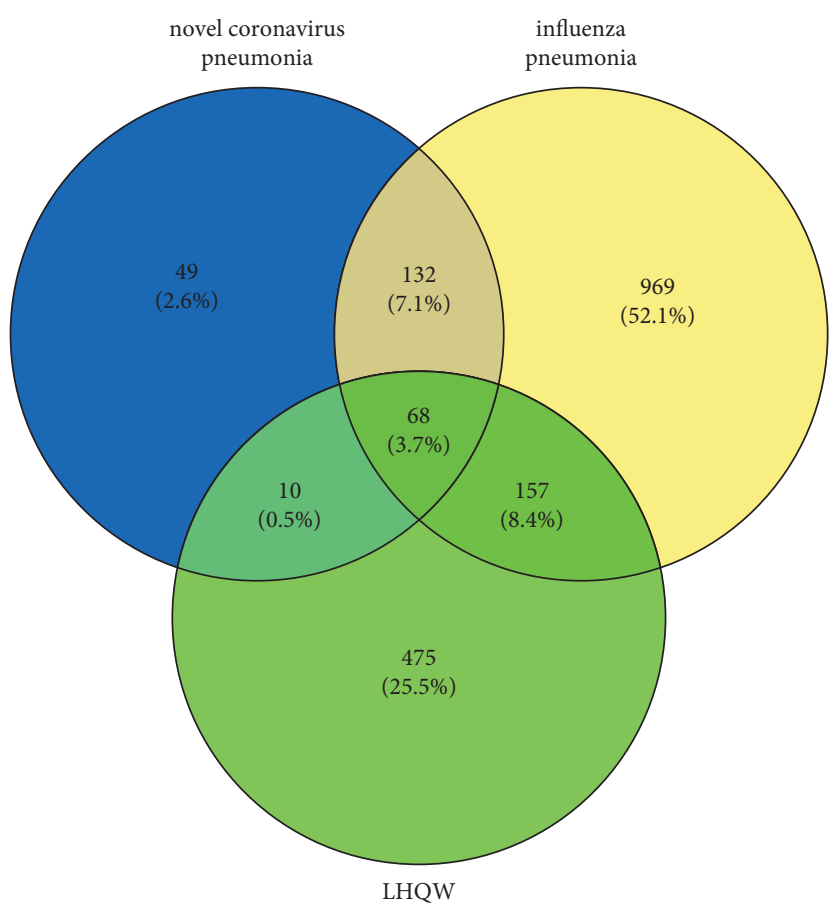

FIGURE 2: Venny figure.

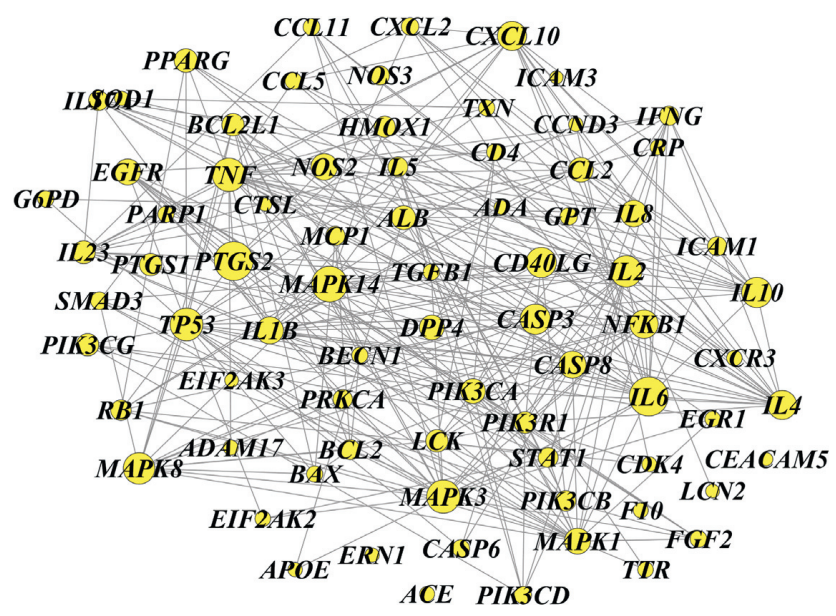

Figure 3: PPI network of LHQW against viral pneumonia. Novel coronavirus pneumonia target (purple), influenza pneumonia target (yellow), and LHQW component target (green).

can cause fever and interfere with apoptosis. As an early cytokine of viral pneumonia, the expression of TNF- $\alpha$ can regulate other proinflammatory factors downstream. An increase in other cytokines stimulates the production of TNF- $\alpha$ and triggers the cytokines that are dependent on TNF- $\alpha$. IL- 6 and TNF- $\alpha$ are the key cytokines $[54,55]$ for the development of IVP and NCP. TNF- $\alpha$, as an early cytokine, can regulate other proinflammatory factors downstream. An increase in other cytokines stimulates the production of both and initiates the TNF- $\alpha$-dependent cytokine cascade reaction, thus forming a vicious spiral that can cause a cytokine storm and aggravate the patient's condition. The activation and inhibition of these targets not only aggravate the disease but also promote each other, resulting in a vicious cycle that is closely related to the inflammatory injury of the lung; it is also the key target of this study. Therefore, an in-depth study of these targets can help reveal the scientific nature and connotation of the treatment of IVP and NCP.

According to the KEGG pathway enrichment analysis in this study, the IL-17 pathway is the most important signal pathway. IL-17 signal pathways are important to the study of multiple coronaviruses. For example, SARS viruses induce the activation of pattern recognition receptors and IL-17 pathways [56]. The Middle East respiratory syndrome coronavirus (MERS-CoV) is considered a highly pathogenic virus infecting the respiratory tract with high morbidity and 


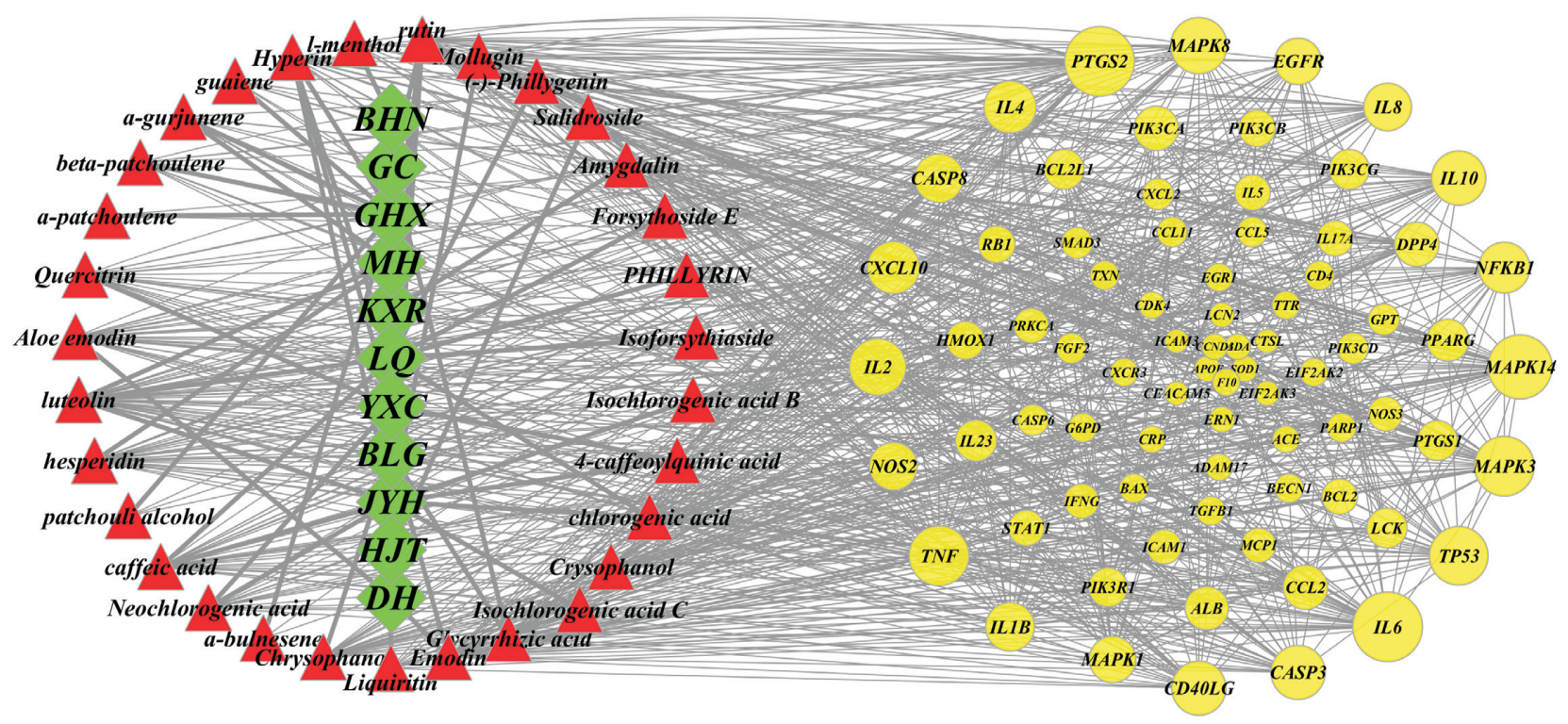

FIGURE 4: "Herb-component-target" network of LHQW in the treatment of viral pneumonia.

TABLE 3: Topological parameter information table for targets.

\begin{tabular}{|c|c|c|c|}
\hline Protein name & Gene name & $\mathrm{BC}$ & $\mathrm{DG}$ \\
\hline Prostaglandin G/H synthase 2 & PTGS2/COX2 & 0.032 & 43 \\
\hline Interleukin-6 & IL-6 & 0.067 & 43 \\
\hline Mitogen-activated protein kinase 14 & MAPK14 & 0.019 & 38 \\
\hline Tumor necrosis factor & TNF- $\alpha$ & 0.043 & 34 \\
\hline Mitogen-activated protein kinase 3 & MAPK3 & 0.046 & 34 \\
\hline Cellular tumor antigen p53 & TP53 & 0.031 & 33 \\
\hline Interleukin-2 & IL2 & 0.020 & 31 \\
\hline Mitogen-activated protein kinase 8 & MAPK8 & 0.022 & 31 \\
\hline Interleukin-10 & IL10 & 0.020 & 29 \\
\hline Caspase-3 & CASP3 & 0.032 & 29 \\
\hline CD40ligand & CD40LG & 0.032 & 29 \\
\hline C-X-C motif chemokine 10 & CXCL10 & 0.022 & 27 \\
\hline Nuclear factor NF-kappa-B1 & $\mathrm{NF}-\kappa \mathrm{B} 1$ & 0.023 & 26 \\
\hline Interleukin-4 & IL4 & 0.009 & 25 \\
\hline Interleukin-1beta & $\operatorname{IL} 1 \beta$ & 0.008 & 24 \\
\hline Caspase- 8 & CASP8 & 0.017 & 24 \\
\hline Nitric oxide synthase, inducible & NOS2 & 0.014 & 23 \\
\hline Interleukin-8 & IL8 & 0.033 & 23 \\
\hline Mitogen-activated protein kinase 1 & MAPK1 & 0.016 & 22 \\
\hline Epidermal growth factor receptor & EGFR & 0.013 & 22 \\
\hline
\end{tabular}

mortality. Animal model studies have shown that MERS$\mathrm{CoV}$ infection can cause a strong inflammatory response. After treatment, inflammatory factors, such as TNF- $\alpha$, IL-2, IL-4, IL-15, and IL-17, can be inhibited relatively. IL-17 signalling pathways regulate IL-17 family expression (first discovered by Th17 cells), and by binding to different receptors, they activate downstream signalling pathways JAK-STAT and NFKB to regulate the secretion of other inflammatory factors; IL-17 signalling pathways are also involved in the occurrence and development of lung injury $[57,58]$. In recent years, in the outbreaks of the flu viruses and SARS and MERS that killed hundreds of millions of patients, excessive inflammation was observed in patients. Related studies have reported that cytokine storm is the most important and direct cause of lung injury. Another work found that the immune system of patients infected with COVID-19 also exhibits a serious cytokine storm phenomenon [59]. Several studies have confirmed that, in the important pathogenesis of host immune response disorders, Th17/Treg immune imbalance can be a major cause $[60,61]$, and Th17/Treg immune imbalance is closely related to cytokine storm. Th17 is a proinflammatory cell. The initiation of Th17 differentiation depends on TGF- $\beta$ and IL-6, which can activate signal transduction and the transcription 


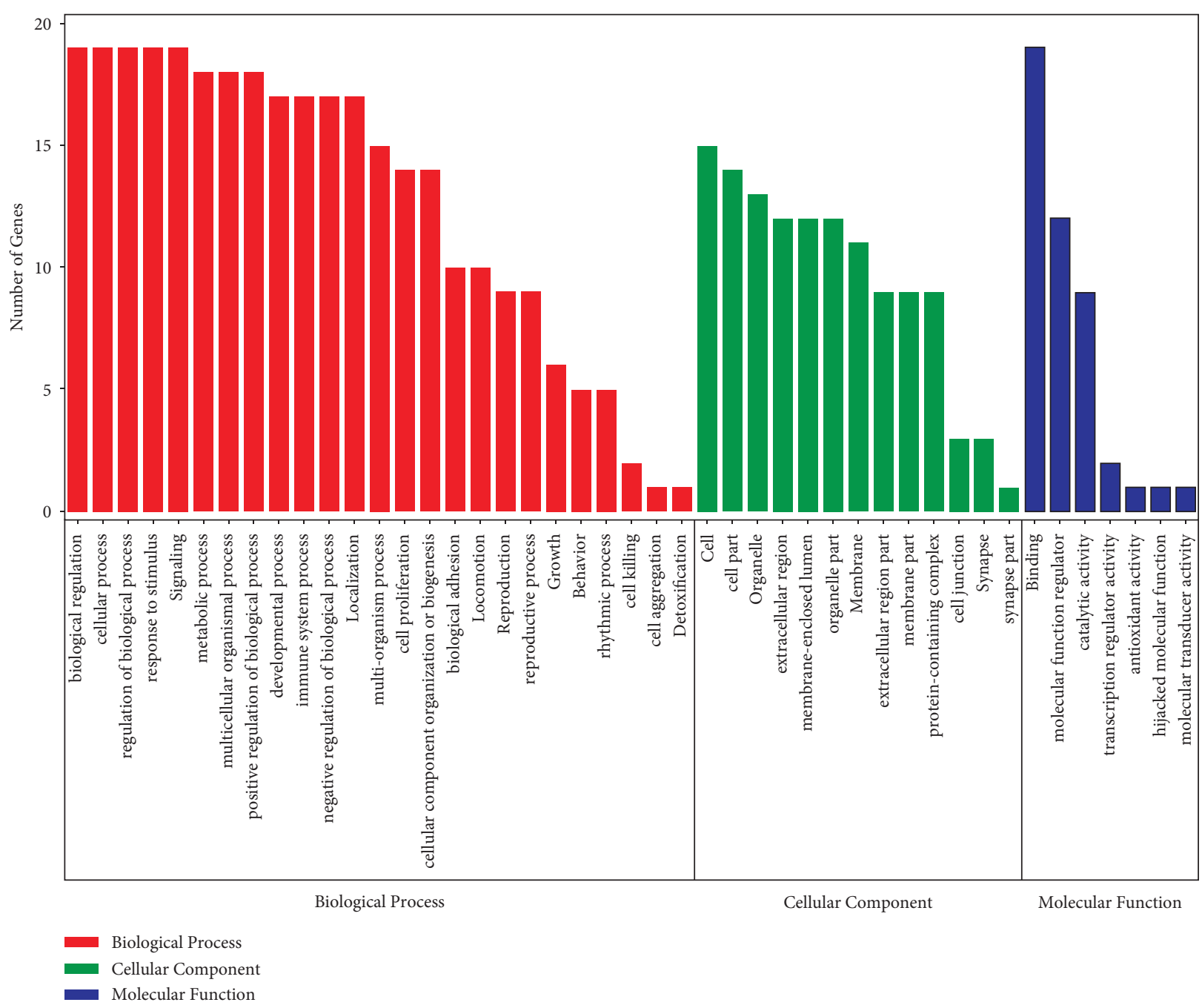

FIgURE 5: GO biofunctional notes. Biological process (red), cellular component (green), and molecular function (blue).

protein pathway by inducing the expression of transcription factors. Th17 mediates inflammatory responses mainly by secreting IL-17, IL-21, IL-2, IL-4, TNF- $\alpha$, and other cytokines $[62,63]$, but an excessive inflammatory response can cause inflammatory pathological damage to the body. TLR family I transmembrane proteins, which are widely distributed in various cells, can recognise a variety of viruses, mediate the body's antiviral innate immunity and promote the development of acquired immunity; this pathway plays an important role in linking immune and acquired immunity [64]. NF- $\kappa \mathrm{B}$ is located at the hub of the downstream signalling pathway in TLR [65]. It can participate in the initiation and regulation of gene expression of many cytokines and inflammatory mediators. After TLR4 binds to its ligand, activated transcription factors enter the nucleus and enhance the transcription of TNF- $\alpha$, IL-1, IL-6, IL- 8 , and other inflammatory factors; they can also upregulate the expression of COX2 and NOS2, which can induce the synthesis of inflammatory mediators (PGE2 and NO). The accumulation of these cytokines can directly reflect the degree of inflammation. A moderate inflammatory response is a kind of defence reaction of the body, and it is conducive to the clearance of pathogens and the recovery of the body. However, an excessive reaction can cause cascading inflammatory reactions that are difficult to control. TLR and $\mathrm{NF}-\kappa \mathrm{B}$ may form a complex cycle network with the participation of certain cytokines, which can regulate each other and form a vicious cycle. Therefore, the 20 intervening key targets and their pathways are key to the treatment of viral pneumonia with LHQW.

In addition, the results of molecular docking showed that the 31 main components contained in LHQW key targets had different degrees of combination. In particular, flavonoids and their glycosides and phenylpropanoids had the best binding result. The varied components contained in LHQW could affect multiple pathways by acting on multiple targets to achieve overall intervention in the development of viral pneumonia and overall treatment. Previous studies have found that chlorogenic acid [66], cryptochlorogenic acid [67], forsythin A [68, 69], phillygenin [70], luteolin [71], salidroside [72, 73], and chrysophanol [74] have significant anti-inflammatory effects. The main mechanism is through the significant inhibition of the activation of NF- $\kappa \mathrm{B}$, TLR, MAPK, and other signalling pathways and downregulation of the gene expression of COX2 


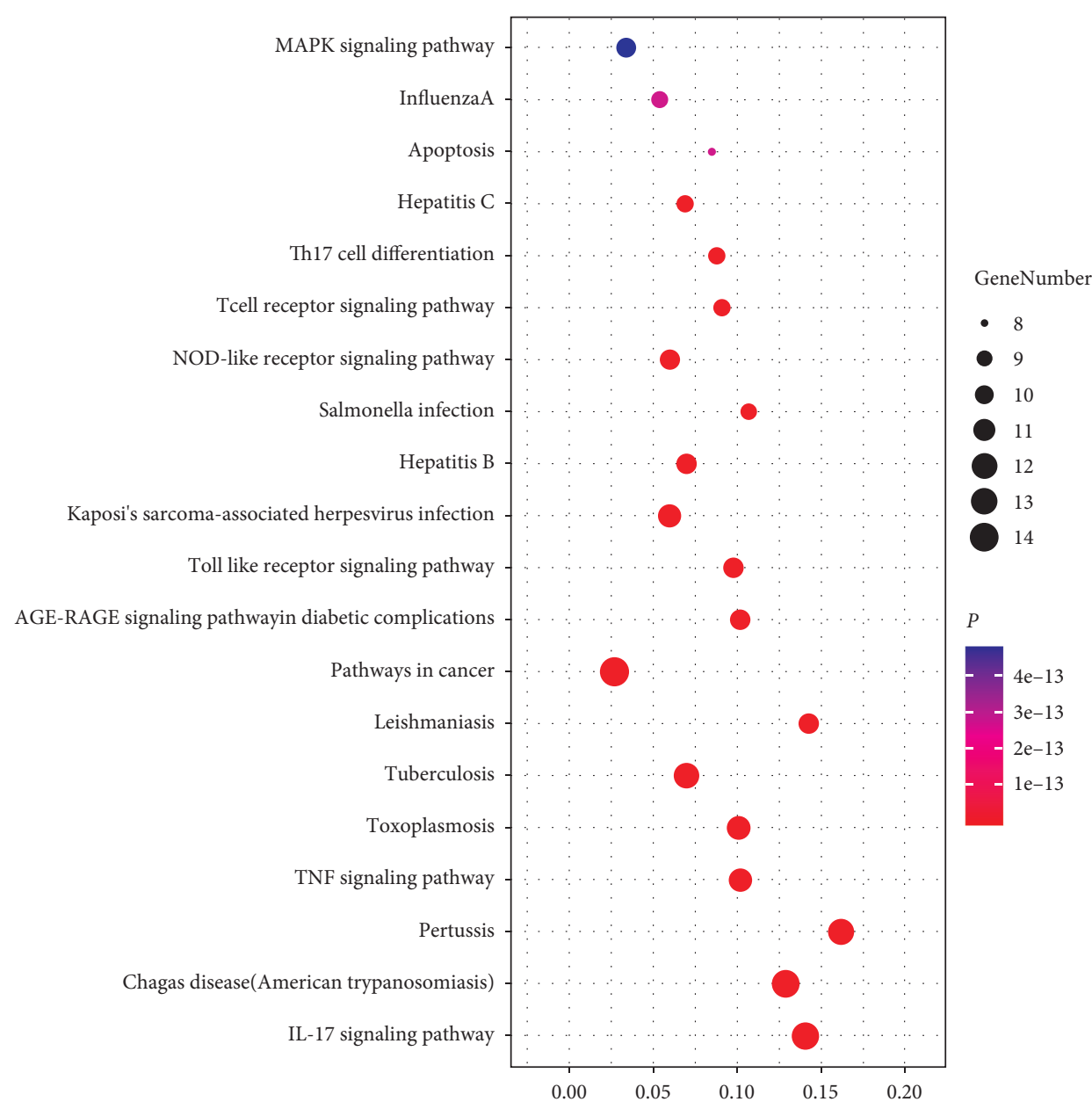

FIGURE 6: KEGG pathway map. The larger the bubble is, the more the targets enriched in the pathway are; the darker the bubble is, the more significant the enrichment pathway is.

and iNOS. As a result, the production of proinflammatory factors, such as IL-6, TNF- $\alpha$, and chemokines (MCP-1, CCL2, and CCL10), is reduced to intervene in acute lung injury. Luteolin $[75,76]$ has a strong inhibitory effect on the proliferation of the influenza virus, but it can also effectively inhibit the expression of inflammatory cytokines and affect the TLR/NF- $\kappa \mathrm{B}$ signalling pathway and the expression of apoptosis-related factors (CASP3/8/9, etc.) to reduce the immune damage on the host cells caused by the virus. It also regulates cell apoptosis and plays an antiviral role. Patchouli alcohol [77] has antibacterial and antiviral effects, and it can significantly inhibit the activities of NA and HA, improve the survival rate of mice infected with $\mathrm{IAV}$, and alleviate the symptoms of pneumonia. Its effect is equivalent to oseltamivir. Its mechanism of action is related to the inhibition of the activation of intracellular PI3K/Akt and ERK/MAPK signalling pathways. Chlorogenic acid and its derivatives, such as isochlorogenic acid $\mathrm{B}$ and $\mathrm{C}[78,79]$, can effectively inhibit influenza virus infection. Their mechanism of action is to downregulate the expression of viral nucleoprotein and inhibit the activity of NA. They can also effectively reduce the inflammatory reaction and inhibit the production of viral infection. The mechanism is to inhibit the TLR signalling pathway in infected cells and reduce the release of inflammatory factors TNF- $\alpha$ and IL- 6 . Forsythia A [80] can inhibit the replication of the influenza virus, control influenza virus infection, and improve the prognosis. Its mechanism is achieved by inhibiting the activation of TLR, NF- $\kappa \mathrm{B}$, and Th17 signalling pathways. Phillyrin [81] can significantly inhibit the replication of SARS-CoV-2 in vitro and the expression of inflammatory cytokines (TNF- $\alpha$, IL- 6, IL-1 $\beta$, CXCL10, etc.) induced by SARS-CoV-2 by regulating the activity of the NF- $\kappa \mathrm{B}$ signalling pathway. Therefore, forsythin can be used as a new strategy for coronavirus disease. Research has confirmed that glycyrrhizic acid [82] is similar to a hormone in chemical structure and has a corticoid effect, and it is mainly used to fight a viral inflammatory storm. Forsythin and glycyrrhizic acid are anti-inflammatory drugs. The main difference between them is that glycyrrhizic acid has no immunosuppressive effect. Therefore, glycyrrhizic acid within the safe dose range can resist an inflammatory storm and does not interfere with the ability of the body to resist viruses. 


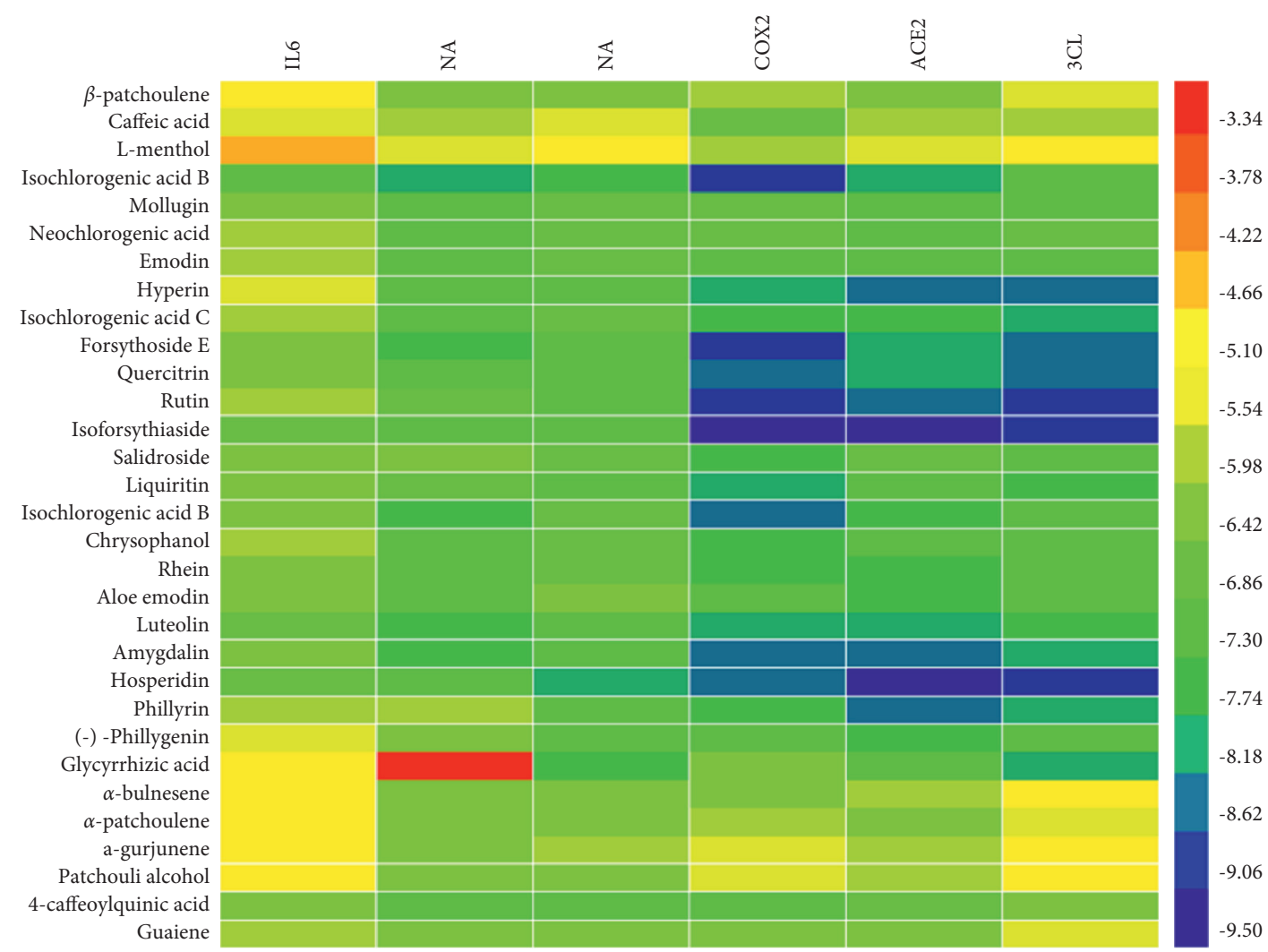

Figure 7: Heatmap of binding energy. The scale on the right represents binding energy, and the colour from red to blue represents the binding energy value arranged from large to small.

Several studies [83] have found that chrysophanol can inhibit the production of IL-2 by B cells and the expression of the CD40 ligand (CD40L) in activated T cells, thus blocking the NF- $\kappa \mathrm{B}$ and MAPK signalling pathways in activated $T$ cells and inhibiting the continuous burst of cytokines. Most of the components reported in these studies are the main components of honeysuckle and forsythia suspense, which belong to the monarch medicine of the LHQW recipe and are the most important components in the treatment of diseases. They clear away heat, detoxify the body, and disperse wind heat. The results of molecular pairing in this study also showed that the main components of honeysuckle and forsythia account for the largest number and are the key to the efficacy of LHQW. The ingredients reported in the above-mentioned studies are basically consistent with the results of the current study, suggesting that the material basis analysis results of this study conform to the law of the monarch, the subject, and TCM to a certain extent. Presently, most studies focus on nonvolatile components, and the few reports on antiviral, anti-inflammatory, and immune regulation of the volatile components of LHQW focus on cooling, expectorant, and antibacterial effects and nasal congestion reduction [84]. The results of molecular docking showed that most of the components of LHQW did not match the new target of IVP and NCP. These results suggest that the contribution of this component to the efficacy of LHQW may not be due to the anti-influenza virus, COVID-19, and antilung inflammation. Volatile components are mainly used to improve nasal congestion, runny nose, cough, expectoration, pharynx, and larynx to create favourable conditions and improve the compliance of patients. All of these compounds are the major components of LHQW and have been extensively studied in the field of influenza infection. Although most of the compounds are not related to NCP, they show good anti-inflammatory, antiviral, and immunoregulation effects, which are closely related to the strategy of treating new crown pneumonia. As the material basis of LHQW, each component exerts a synergistic effect together with the interaction of each target, and these ingredients contribute to the efficacy of LHQW.

An abnormal immune system is the main common point in the occurrence and development of IVP and NCP. The pathway enrichment analysis and biological function annotation in this study showed that the mechanism of LHQW in the treatment of the two diseases is closely related to immune, inflammatory, and viral signalling pathways. Therefore, the mechanism of LHQW treating IVP and NCP could focus on restoring the physiological function of the immune system, reducing the persistent injury of inflammation to the body, inhibiting the invasion and proliferation of the virus, and promoting the apoptosis of the virus. 


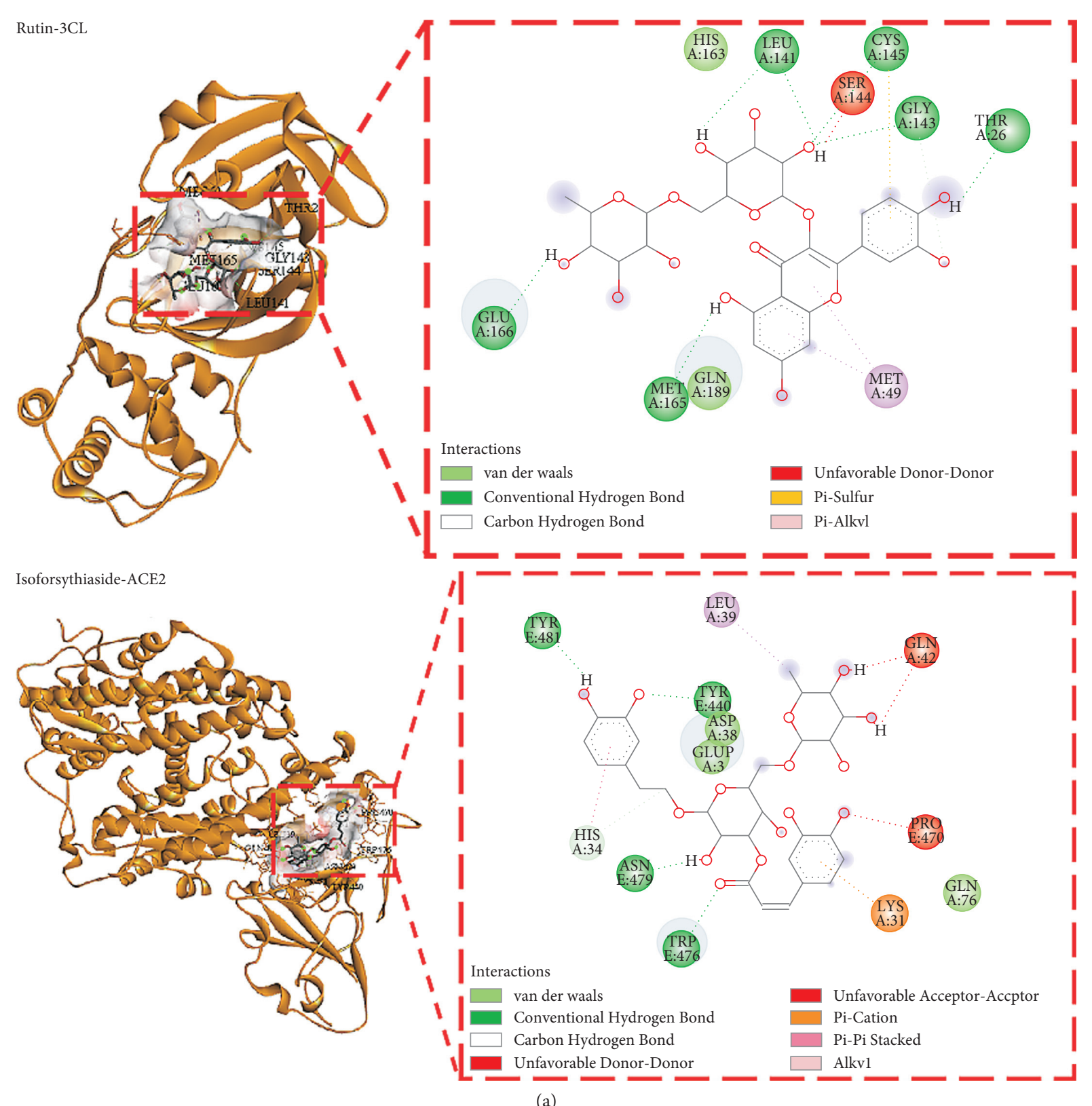

Figure 8: Continued. 


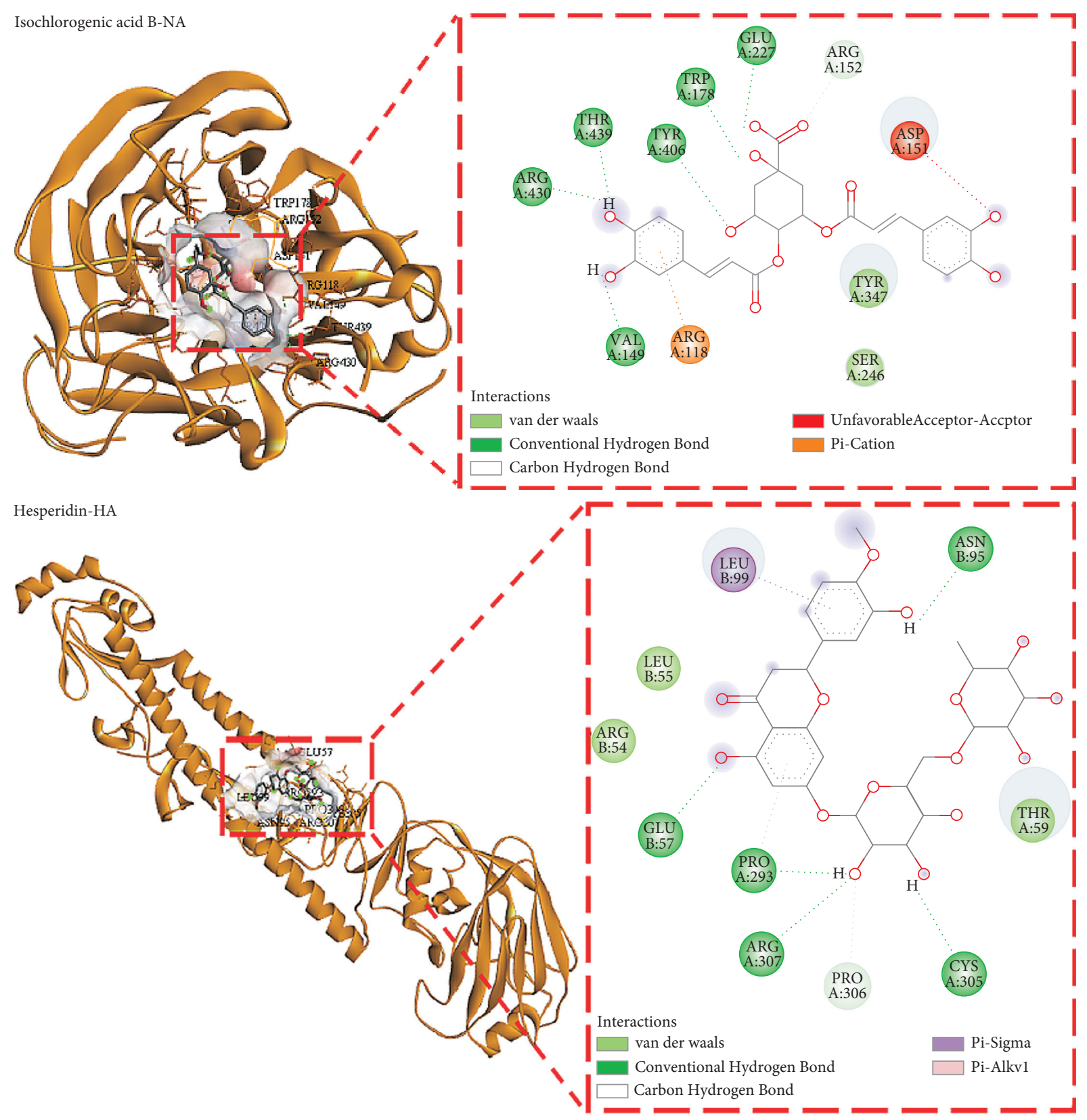

(b)

Figure 8: Continued. 


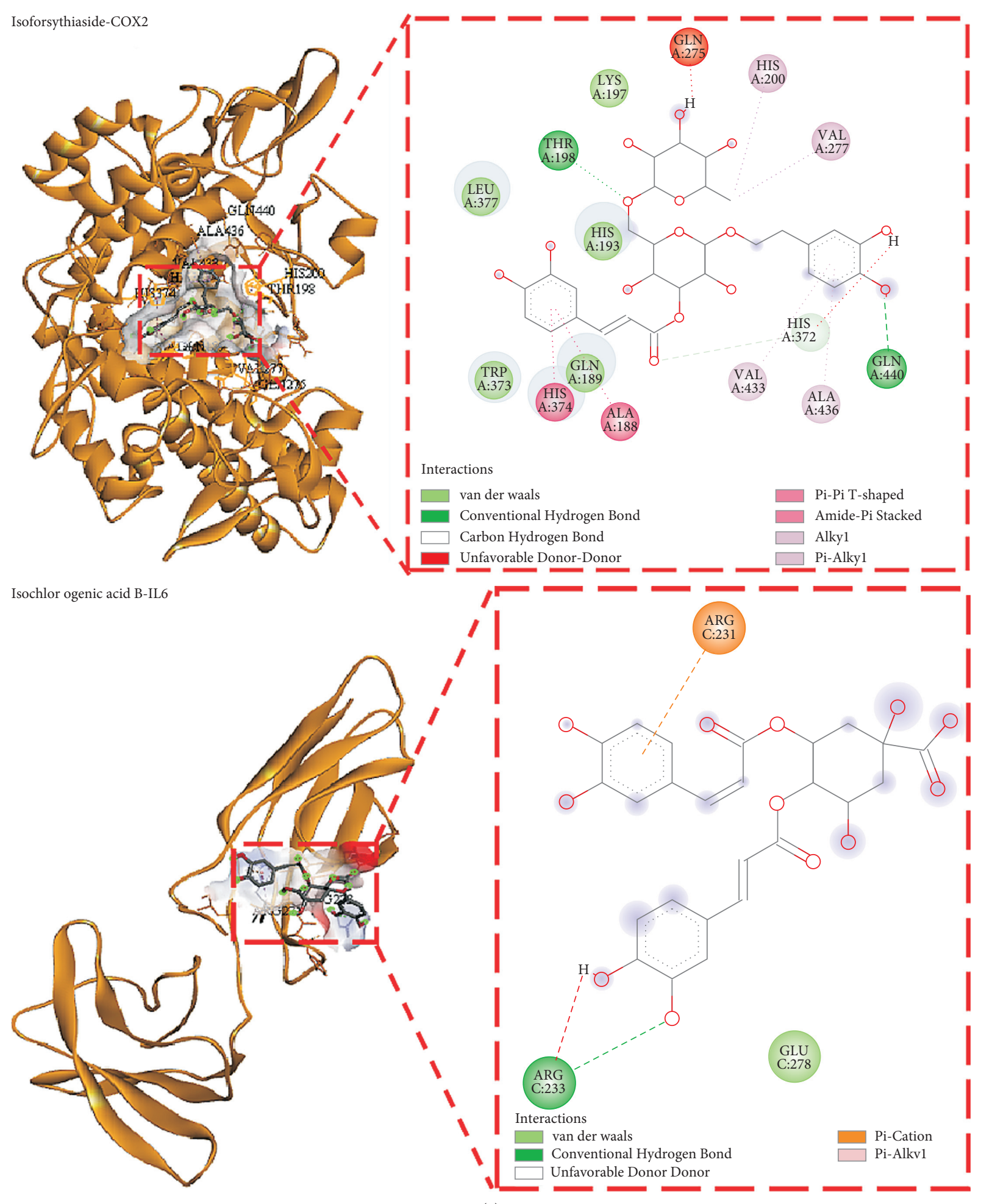

(c)

FIGURE 8: Interactions between the top five components of LHQW and the amino acids of targets related to IVP and NCP with binding energy less than $-7.0 \mathrm{kcal} / \mathrm{mol}$. (a) Main components of anti-NCP. (b) Main components of anti-IVP. (c) Main consensus components of anti-IVP and anti-NCP. 
TABLE 4: Normalisation and comprehensive evaluation index of molecular docking results.

\begin{tabular}{|c|c|c|c|c|c|c|c|c|}
\hline Serial number & Molecular & $3 \mathrm{CL}$ & ACE2 & $\begin{array}{c}\text { COX2 } \\
\text { Convergence }\end{array}$ & $\begin{array}{c}\text { HA } \\
\text { values }\end{array}$ & IL-6 & NA & Composite index \\
\hline 1 & Isoforsythiaside & 0.95 & 1.00 & 1.00 & 0.69 & 0.92 & 0.86 & 0.90 \\
\hline 2 & Hesperidin & 0.95 & 0.97 & 0.77 & 1.00 & 0.92 & 0.84 & 0.89 \\
\hline 3 & Isochlorogenic acid B & 0.58 & 0.68 & 0.81 & 0.91 & 1.00 & 1.00 & 0.81 \\
\hline 4 & Forsythoside E & 0.86 & 0.62 & 0.81 & 0.78 & 0.71 & 0.90 & 0.78 \\
\hline 5 & Quercitrin & 0.88 & 0.65 & 0.74 & 0.72 & 0.75 & 0.88 & 0.77 \\
\hline 6 & Amygdalin & 0.79 & 0.73 & 0.77 & 0.75 & 0.63 & 0.94 & 0.76 \\
\hline 7 & Rutin & 1.00 & 0.76 & 0.88 & 0.72 & 0.42 & 0.78 & 0.73 \\
\hline 8 & Luteolin & 0.63 & 0.62 & 0.63 & 0.75 & 0.88 & 0.90 & 0.72 \\
\hline 9 & Isochlorogenic acid $\mathrm{C}$ & 0.79 & 0.57 & 0.5 & 0.63 & 0.86 & 0.65 & 0.66 \\
\hline 10 & Hyperin & 0.86 & 0.73 & 0.60 & 0.69 & 0.38 & 0.80 & 0.65 \\
\hline 11 & Chlorogenic acid & 0.56 & 0.57 & 0.70 & 0.56 & 0.67 & 0.90 & 0.65 \\
\hline 12 & DFV & 0.67 & 0.43 & 0.67 & 0.72 & 0.63 & 0.76 & 0.64 \\
\hline 13 & Rhein & 0.58 & 0.57 & 0.58 & 0.56 & 0.67 & 0.86 & 0.63 \\
\hline 14 & Phillyrin & 0.72 & 0.84 & 0.56 & 0.66 & 0.46 & 0.60 & 0.63 \\
\hline 15 & Chrysophanol & 0.51 & 0.49 & 0.56 & 0.56 & 0.46 & 0.88 & 0.56 \\
\hline 16 & Aloe emodin & 0.51 & 0.54 & 0.49 & 0.50 & 0.58 & 0.80 & 0.56 \\
\hline 17 & Emodin & 0.53 & 0.49 & 0.40 & 0.63 & 0.54 & 0.84 & 0.56 \\
\hline 18 & Mollugin & 0.56 & 0.46 & 0.35 & 0.59 & 0.58 & 0.86 & 0.55 \\
\hline 19 & Caffeoylquinic acid 4 & 0.40 & 0.30 & 0.49 & 0.78 & 0.67 & 0.84 & 0.54 \\
\hline 20 & Salidroside & 0.53 & 0.30 & 0.53 & 0.53 & 0.67 & 0.68 & 0.52 \\
\hline 21 & Neochlorogenic acid & 0.47 & 0.46 & 0.37 & 0.59 & 0.50 & 0.86 & 0.52 \\
\hline 22 & (-)-Phillygenin & 0.51 & 0.57 & 0.40 & 0.72 & 0.38 & 0.64 & 0.52 \\
\hline 23 & Guaiene & 0.14 & 0.16 & 0.26 & 0.47 & 0.42 & 0.70 & 0.30 \\
\hline 24 & Caffeic acid & 0.21 & 0.08 & 0.37 & 0.13 & 0.33 & 0.58 & 0.23 \\
\hline 25 & $\alpha$-Patchoulene & 0.12 & 0.14 & 0.12 & 0.44 & 0.21 & 0.68 & 0.22 \\
\hline 26 & $\beta$-Patchoulene & 0.12 & 0.14 & 0.12 & 0.44 & 0.21 & 0.68 & 0.22 \\
\hline 27 & $\alpha$-Bulnesene & 0.07 & 0.08 & 0.19 & 0.50 & 0.13 & 0.70 & 0.19 \\
\hline 28 & $\alpha$-Gurjunene & 0.07 & 0.03 & 0.07 & 0.31 & 0.04 & 0.66 & 0.10 \\
\hline 29 & Patchouli alcohol & 0.09 & 0.11 & 0.00 & 0.38 & 0.08 & 0.64 & 0.00 \\
\hline 30 & Glycyrrhizic acid & 0.74 & 0.43 & 0.21 & 0.88 & 0.17 & 0.00 & 0.00 \\
\hline 31 & L-menthol & 0.00 & 0.00 & 0.16 & 0.00 & 0.00 & 0.46 & 0.00 \\
\hline
\end{tabular}

\section{Conclusion}

LHQW contains 31 main components. Isochlorogenic acid B, isoforsythiaside, forsythoside E, rutin, quercitrin, hesperidin, and other components are the key to the simultaneous treatment of NCP and IVP by LHQW. These components can interfere with signalling pathways, such as IL-17, T cell receptor, Th17 cell differentiation, TNF, toll-like receptor, MAPK, and apoptosis by acting on targets, including 3CL, ACE2, COX2, HA, IL-6, and NA, to realise the anti-inflammatory, antivirus, and immune regulation comprehensive efficacy of LHQW. Although the mechanism of treating IVP and NCP differs and the different contents are mainly reflected in the manner of virus infection and the material basis of antivirus, the main links are closely related to regulating immunity, reducing inflammation, and combating the virus. The process embodies the characteristics of seeking common ground whilst reserving differences in the treatment process of IVP and NCP. It also shows the multicomponent, multitarget, and multichannel advantages of LHQW and provides a research method for the mechanism and substance foundation of the treatment of IVP and NCP.

\section{Data Availability}

The original data used to support the findings of this study are included within the supplementary information file.

\section{Conflicts of Interest}

The authors declare no conflicts of interest.

\section{Authors' Contributions}

Huihui Su and Guosong Wu contributed equally to the work. Huihui Su and Guosong $\mathrm{Wu}$ formulated the idea of the paper. Huihui Su, Guosong Wu, Lulu Zhan, and Fei Xu performed the research, analysed the data, and wrote the paper. Huihui Su, Huiqin Qian, and Yanling Li participated in revising the data and improving paper writing. Ximei Zhu 
supervised the research. All authors reviewed the paper, and all authors read and approved the final version of the paper.

\section{Acknowledgments}

This work was carried out with the support of the Universitylevel application project of Xinxiang Medical University (grant/award no. XJKT201920); Program for Training Key Teachers of Sanquan College of Xinxiang Medical University (grant/award no. SQ2021GGJS05), and Science and Technology Bureau Project of Huadu District (grant/award no. 21-HDWS-109).

\section{Supplementary Materials}

Supplementary Table 1: molecular docking results. Supplementary Table 2: the top five components with binding energy less than $-7.0 \mathrm{kcal} / \mathrm{mol}$. Supplementary Figure 3: interactions between the top five components of LHQW with the amino acids of targets related to IVP and NCP with binding energy less than $-7.0 \mathrm{kcal} / \mathrm{mol}$. . (Supplementary Materials)

\section{References}

[1] J. J. Zhu, "Clinical application and research progress of Lianhua Qingwen preparation," Strait Pharmaceutical Journal, vol. 31, no. 11, pp. 200-202, 2019.

[2] K. T. Yao, M. Y. Liu, X. Li, X. Q. Huang, and H. B. Cai, "Retrospective clinical study on novel coronavirus pneumonia treated with Lianhua Qingwen," Chinese Journal of Experimental Traditional Medical Formulae, vol. 26, no. 11, pp. 8-12, 2020.

[3] A. A. Ishaqui, A. H. Khan, S. A. Sulaiman, M. Alsultan, and I. Khan, "Efficacy comparison of oseltamivir alone and oseltamivir-antibiotic combination for early resolution of symptoms of severe influenza-A and influenza-B hospitalized patients," Saudi Medical Journal, vol. 41, no. 9, pp. 1015-1021, 2020.

[4] D. Z. Cheng, W. J. Wang, Y. Li, X. D. Wu, B. Zhou, and Q. Y. Song, "Novel coronavirus pneumonitis patients treated with Chinese herbal medicine Lianhua Qingwen: a multicenter retrospective study of 51 cases," Tianjin Journal of Traditional Chinese Medicine, vol. 37, no. 5, pp. 509-516, 2020.

[5] R. B. Lv, W. J. Wang, and X. Li, "Novel coronavirus pneumonia suspected cases treated with Lianhua Qingwen Decoction: a clinical observation of 63 cases," Journal of Traditional Chinese Medicine, vol. 16, pp. 1-5, 2020.

[6] H. R. Li, L. P. Chang, C. Rui, and Z. H. Jia, "Novel coronavirus pneumonia: a theoretical basis and clinical efficacy of Lianhua Qingwen," World Chinese Medicine, vol. 15, no. 3, pp. 332336, 2020.

[7] H. Q. Zhang and S. Li, "Advances in network pharmacology and modern research of traditional Chinese Medicine," Chinese Journal of Pharmacology and Toxicology, vol. 29, no. 6, pp. 883-892, 2015.

[8] L. L. Wang, B. Li, S. Y. Wang, and J. Zhang, "Application progress of system biology technology in traditional Chinese medicine research based on holistic view," Chinese Traditional and Herbal Drugs, vol. 51, no. 19, pp. 5053-5064, 2020.

[9] Y. Ren, Y. J. Deng, and H. B. Ma, "Research progress and challenges of network pharmacology in the field of traditional Chinese Medicine," Chinese Traditional and Herbal Drugs, vol. 51, no. 18, pp. 4789-4797, 2020.
[10] H. H. Xu, S. M. Li, R. Xu, L. Fang, H. Xu, and P. J. Tong, "Predication of the underlying mechanism of Bushenhuoxue formula acting on knee osteoarthritis via network pharmacology-based analyses combined with experimental validation," Journal of Ethnopharmacology, vol. 263, 2020.

[11] T. H. Liu, W. Q. Tu, W. C. Tao, Q. E. Liang, Y. Xiao, and L. G. Chen, "Verification of resveratrol inhibits intestinal aging by downregulating ATF4/chop/bcl-2/bax signaling pathway: based on network pharmacology and animal experiment," Frontiers in Pharmacology, vol. 11, p. 1064, 2020.

[12] C. Zhang, D. Guan, M. Jiang et al., "Efficacy of leflunomide combined with ligustrazine in the treatment of rheumatoid arthritis: prediction with network pharmacology and validation in a clinical trial," Chinese Medicine, vol. 14, no. 1, p. 26, 2019.

[13] J. S. Tian and X. M. Qin, "Application of network pharmacology in the research of "treating the same disease with different methods" and "treating different diseases with the same method"," Chinese Journal of Pharmacology and Toxicology, vol. 32, no. 11, pp. 861-862, 2018.

[14] X. P. Zhao, W. R. Yu, and X. Li, "Establishment pharmacological research platform for "Concurrent treatment of the brain and heart" and its application on dissecting mechanism of Danhong injection," China Journal of Chinese Materia Medica, vol. 42, no. 24, pp. 4905-4912, 2017.

[15] H. Y. Zhu, H. P. Li, and X. Gao, "Network pharmacological mechanism of Jiaotai pill in treating diabetes, depression and insomnia," Modernization of Traditional Chinese Medicine and Materia medica-World Science and Technology, vol. 20, no. 3, pp. 460-467, 2018.

[16] X. J. Wang, W. T. Zhang, M. R. Wang, Q. Y. Li, and W. L. Weng, "Study on mechanism of treating different diseases with same method of Shengmai decoction in treating diabetes and heart failure based on network pharmacology," Chinese Journal of Information on Traditional Chinese-Medicine, vol. 28, no. 1, pp. 19-26, 2021.

[17] K. B. Sun and R. Sun, "Network pharmacological analysis of Chaihu Guizhi Decoction for treating different diseases with same method on gastric ulcer and epilepsy," Chinese Traditional and Herbal Drugs, vol. 50, no. 21, pp. 5178-5186, 2019.

[18] G. Stelzer, N. Rosen, I. Plaschkes et al., "The GeneCards suite: from gene data mining to disease genome sequence analyses," Current Protocols in Bioinformatics, vol. 54, no. 1, pp. 1-33, 2016.

[19] R. Raúl, M. Yamina, and R. Udi, "Identification of relevant genetic alterations in cancer using topological data analysis," Nature Communications, vol. 11, no. 1, 2020.

[20] "Information technology-data analytics; new findings from university of salamanca in the area of data analytics reported (centrality measures in simplicial complexes: applications of topological data analysis to network science)," 2020, https:// arxiv.org/abs/1908.02583.

[21] S. Liu, X. Hu, X. Fan et al., "A bioinformatics research on novel mechanism of compound kushen injection for treating breast cancer by network pharmacology and molecular docking verification," Evidence-based Complementary and Alternative Medicine: eCAM, vol. 2020, Article ID 2758640, 14 pages, 2020.

[22] K. F. Taha, M. Khalil, M. S. Abubakr, and E. Shawky, "Identifying cancer-related molecular targets of Nandina domestica Thunb. by network pharmacology-based analysis in combination with chemical profiling and molecular docking studies," Journal of Ethnopharmacology, vol. 249, p. 112413, 2020. 
[23] H. Yu, W. N. Jia, J. P. Liu, Y. Zhu, and C. H. Wang, “Analysis of blood components in Lianhua Qingwen capsule by UPLCQ-TOF-MS," Tianjin Journal of Traditional Chinese Medicine, vol. 33, no. 12, pp. 756-759, 2016.

[24] W. N. Jia, C. H. Wang, and Y. F. Wang, "Qualitative and quantitative analysis of the major constituents in Chinese medical preparation Lianhua-Qingwen capsule by UPLCDAD-QTOF-MS," The Scientific World Journal, vol. 2015, Article ID 731765, 19 pages, 2015.

[25] Q. F. Qin, "Determination of chlorogenic acid, forsythin, rhein, emodin and chrysophanol in lianhuan qingwen capsules by HPLC," Chinese Journal of Modern Applied Pharmacy, vol. 31, no. 2, pp. 210-213, 2014.

[26] Y. F. Zhang, M. Y. Liu, Z. J. Li, H. H. Xu, and Y. L. Bai, "Rapid determination of ingredients in water extract of Lianhua qingwen capsule by UPLC," Chinese Journal of Modern Applied Pharmacy, vol. 31, no. 01, pp. 78-80, 2014.

[27] G. G. P. Bruna, E. R. O. Antonio, and S. Youvika, "ACE2 expression is increased in the lungs of patients with comorbidities associated with severe COVID-19," The Journal of Infectious Diseases, vol. 222, no. 4, 2020.

[28] K. Ibrahim, N. Asad, S. Remah, and B. Hassan, "Polyacylated anthocyanins constructively network with catalytic dyad residues of $3 \mathrm{CL}^{\text {pro }}$ of $2019-\mathrm{nCoV}$ than monomeric anthocyanins: a structural-relationship activity study with 10 anthocyanins using in-silico approaches," Journal of Molecular Graphics \& Modelling, vol. 100, 2020.

[29] M. T. U. Qamar, S. M. Alqahtani, M. A. Alamri, and L. L. Chen, "Structural basis of SARS-CoV-2 3CL (pro) and anti-COVID-19 drug discovery from medicinal plants," Journal of Pharmaceutical Analysis, vol. 10, no. 4, pp. 313-319, 2020.

[30] P. F. N. Souza, F. E. S. Lopes, J. L. Amaral, C. D. T. Freitas, and J. T. A. Oliveira, "A molecular docking study revealed that synthetic peptides induced conformational changes in the structure of SARS-CoV-2 spike glycoprotein, disrupting the interaction with human ACE2 receptor," International Journal of Biological Macromolecules, vol. 164, pp. 66-76, 2020.

[31] R. Yu, L. P. Cheng, M. Li, and W. Pang, "Discovery of novel neuraminidase inhibitors by structure-based virtual screening, structural optimization, and bioassay," ACS Medicinal Chemistry Letters, vol. 10, no. 12, pp. 1667-1673, 2019.

[32] S. Sajjadi, A. Shirode, S. R. Vaidya, and S. S. Cherian, "Molecular mechanism by which residues at position 481 and 546 of measles virus hemagglutinin protein define CD46 receptor binding using a molecular docking approach," Computational Biology and Chemistry, vol. 80, pp. 384-389, 2019.

[33] V. D. W. Roosmarijn and T. H. L. T. Llhan, "Drivers of recombinant soluble influenza $\mathrm{A}$ virus hemagglutinin and neuraminidase expression in mammalian cells," Protein Science: A Publication of the Protein Society, vol. 29, no. 9, 2020.

[34] S. Chen, G. Liu, and J. G. Chen, "Ponatinib protects mice from lethal influenza infection by suppressing cytokine storm," Frontiers in Immunology, vol. 10, 2019.

[35] N. L. Christian and B. Johann, "Extracorporeal hemoadsorption: an option for COVID-19 associated cytokine storm syndrome," Shock, vol. 54, 2020.

[36] W. Wu, G. H. Cui, and B. Lu, "Optimization of multiple evariables:application of central composite design and overall desirability," Chinese Pharmaceutical Journal, vol. 8, pp. 26-29, 2000 .
[37] S. Pirzada, M. N. Uddin, T. D. Figley et al., "Spatial normalization of multiple sclerosis brain MRI data depends on analysis method and software package," Magnetic Resonance Imaging, vol. 68, pp. 83-94, 2020.

[38] Z. Z. Wang, W. Z. Huang, J. P. Qi, W. Wu, and Y. Lu, "Progress on application of central composite design-response surface methodology in optimization of preparations in China," Acta Pharmaceutica Sinica, vol. 12, pp. 1-17, 2020.

[39] P. L. Cheng, L. W. Li, S. J. Zai, and T. Lan, "Study on the extraction technique of poly-methoxyflavonoids from citrus peels by using response surface methodology," Advanced Materials Research, vol. 1919, 2012.

[40] Y. Q. Tao, T. Liang, and K. Y. Zhou, "The role of three metabolic pathways of arachidonic acid in inflammatory response," International Journal of Immunology, vol. 4, pp. 303-306+314, 2010.

[41] F. J. Li, Mechanism of COX2 Gene Expression Induced by Influenza A Virus Infection, Wuhan University, Wuhan, China, 2011.

[42] L. Liu, Z. Y. Cao, J. Chen, and Y. Zhu, "Influenza A virus induces interleukin-27 expression through COX2 and PKA signaling pathways," in Proceedings of the Hubei society of Microbiology, Wuhan society of microbiology Abstracts of 2013 Hubei and Wuhan Microbiology Society Member Congress and Annual Meeting, Wuhan, China, May 2013.

[43] Y. Re, M. C. Yao, and X. Q. Huo, “"Studyon treatment of"cytokine storm" by anti-2019-nCoV prescriptions based on arachidonic acid metabolic pathway," China Journal of Chinese Materia Medica, vol. 45, no. 6, pp. 1225-1231, 2020.

[44] L. J. Ying, "The role of COX-2 in acute lung injury," Chinese Journal of Emergency Medicine, vol. 12, pp. 1341-1342, 2009.

[45] P. Y. Li, "Changes and significance of COX-2 in endotoxin induced acute lung injury in rats," China Practical Medicine, vol. 7, no. 27, pp. 248-249, 2012.

[46] N. Y. Asaad and G. S. Sadek, Pulmonary cryptosporidiosis: Role of COX2 and NF-kB, vol. 114, no. 10, 2006.

[47] L. Wang, X. J. Wang, and Q. Wang, "Effect of COX2 inhibitor on growth and inflammatory response of rat alveolar macrophages and its mechanism," Journal of Clinical and Experimental Medicine, vol. 18, no. 19, pp. 2035-2039, 2019.

[48] C. Li, P. Yang, Y. Zhang et al., "Corticosteroid treatment ameliorates acute lung injury induced by 2009 swine origin influenza A (H1N1) virus in mice," PLoS One, vol. 7, no. 8, p. e44110, 2012.

[49] L. Tang, Z. N. Yin, Y. Hu, and H. Mei, "Controlling cytokine storm is vital in COVID-19," Frontiers in Immunology, vol. 11, 2020.

[50] C. Q. Yang, J. Zhai, and Y. T. Liu, "Immune imbalance mechanism and intervention strategy in patients with coronavirus disease 2019 (COVID-19)," Chinese Pharmacological Bulletin, vol. 36, no. 4, pp. 445-453, 2020.

[51] J. G. X. Zhi and G. T. Paul, "New fronts emerge in the influenza cytokine storm," Seminars in Immunopathology, vol. 39, no. 5, 2017.

[52] D. Ray, S. Jolly, T. Hinton et al., " $\alpha$ Predicting radiation pneumonitis using plasma biomarkers related to tnf $\alpha$-nf?b pathway," International Journal of Radiation Oncology, Biology, Physics, vol. 108, no. 3, p. e570, 2020.

[53] W. Ye, S. Lu, and A. Xue, " $\alpha$ The potential role of TNF $\alpha$ in 2019 novel coronavirus pneumonia," Respiratory Medicine Case Reports, vol. 30, p. 101087, 2020.

[54] P. Eakachai, K. Chutitorn, and P. Tanapat, "Immune responses in COVID-19 and potential vaccines: lessons learned 
from SARS and MERS epidemic," Asian Pacific Journal of Allergy and Immunology, vol. 38, no. 1, 2020.

[55] D. Wang, B. Hu, C. Hu et al., "Clinical characteristics of 138 hospitalized patients with 2019 novel coronavirus-infected pneumonia in wuhan, China," JAMA, vol. 323, no. 11, pp. 1061-1069, 2020.

[56] F. F. Zhai and Y. C. Qian, "New regulatory mechanisms of IL17 signaling pathway," Journal of Shanghai Jiaotong University, vol. 33, no. 4, p. 420, 2013.

[57] S. Cafarotti, "Severe acute respiratory syndrome-coronavirus2 infection and patients with lung cancer: the potential role of interleukin-17 target therapy," Journal of Thoracic Oncology, vol. 15, no. 7, 2020.

[58] Q. Li, Y. Gu, Q. Tu, K. Wang, X. Gu, and T. Ren, "Blockade of interleukin-17 restrains the development of acute lung injury," Scandinavian Journal of Immunology, vol. 83, no. 3, pp. 203-211, 2016.

[59] A. Erhan, Ö. Murat, A. İsa, A. Ayman, and A. Eşref, "Potential role of anti-interleukin-17 in COVID-19 treatment," Dermatologic Therapy, vol. 33, no. 4, 2020.

[60] C. C. Hoogenraad and L. Riol-Blanco, "Interleukin-17: a social cytokine," Cell, vol. 181, no. 3, pp. 517-519, 2020.

[61] Z. Xu, L. Shi, Y. Wang et al., "Pathological findings of COVID19 associated with acute respiratory distress syndrome," Lancet Respiratory Medicine, vol. 8, no. 4, pp. 420-422, 2020.

[62] G. Virella, Medical Immunology, Taylor \& Francis, New York, NY, USA, Sixth edition, 2013.

[63] X. X. Zhang and Z. W. Zeng, "Research progress of imbalance of Th17 cells and Treg cells in chronic inflammatory diseases," Journal of Clinical and Pathological Research, vol. 34, no. 5, pp. 601-605, 2014.

[64] M. Akinnusi, P. Jaoude, T. Kufel, and A. A. El-Solh, "Toll-like receptor activity in patients with obstructive sleep apnea," Sleep \& breathing=Schlaf \& Atmung, vol. 17, no. 3, pp. 1009-16, 2013.

[65] C. Cao, C. Yin, S. Shou et al., "Ulinastatin protects against LPS-induced acute lung injury by attenuating TLR4/NF- $\kappa \mathrm{B}$ pathway activation and reducing inflammatory mediators," Shock, vol. 50, no. 5, pp. 595-605, 2018.

[66] X. Fu, X. Lyu, H. Liu et al., "Chlorogenic acid inhibits BAFF expression in collagen-induced arthritis and human synoviocyte MH7A cells by modulating the activation of the NF$\kappa \mathrm{B}$ signaling pathway," Journal of immunology research, vol. 2019, Article ID 8042097, 10 pages, 2019.

[67] X. L. Zhao, L. Yu, and S. D. Zhang, "Cryptochlorogenic acid attenuates LPS-induced inflammatory response and oxidative stress via upregulation of the $\mathrm{Nrf} 2 / \mathrm{HO}-1$ signaling pathway in RAW 264.7 macrophages," International Immunopharmacology, vol. 83, 2020.

[68] X. Y. Zeng, W. Yuan, L. Zhou, S. X. Wang, Y. Xie, and Y. J. Fu, "Forsythoside A exerts an anti-endotoxin effect by blocking the LPS/TLR4 signaling pathway and inhibiting Tregs in vitro," International Journal of Molecular Medicine, vol. 40, no. 1, pp. 243-250, 2017.

[69] B. L. Zi, S. H. Liu, and J. Y. Ou, "Corrigendum to "Forsythoside A inhibits adhesion and migration of monocytes to type II alveolar epithelial cells in lipopolysaccharide-induced acute lung injury through upregulating miR-124", " Toxicology and Applied Pharmacology, vol. 409, 2020.

[70] R. Q. Tang, Y. Y. Quan, L. H. Yu, L. Zheng, and Y. X. Li, "Effect of Phillygenin on inflammatory response in LPS-induced RAW 264.7 cells," Natural Product Research and Development, vol. 31, no. 7, pp. 1117-1123, 2019.
[71] C. Y. Chen, W. H. Peng, K. D. Tsai, and S. L. Hsu, "Luteolin suppresses inflammation-associated gene expression by blocking NF-kappaB and AP-1 activation pathway in mouse alveolar macrophages," Life Sciences, vol. 81, no. 23-24, pp. 1602-1614, 2007.

[72] J. Wang, Y. L. Zhang, and N. Zhuang, "Salidroside inhibits inflammatory factor release in BV-2 cells through p38 and JNK pathways," Sheng Li Xue Bao: Acta Physiologica Sinica, vol. 70, no. 3, p. 245, 2018.

[73] Y. C. Yuan and L. Hua, "Protective effects of salidroside on lung injury in rats with acute respiratory distress syndrome," The Chinese Journal of Clinical Pharmacology, vol. 36, no. 7, pp. 782-784+788, 2020 .

[74] Q. Guo, Q. S. He, F. R. Hu, Y. D. Cai, and Y. H. Li, "Chrysophanol regulates microglial inflammatory response through TLR4/NF-kB signaling pathway," Medical Information, vol. 33, no. 19, pp. 51-54+58, 2020.

[75] D. D. Yuan, Effects of Luteolin on Influenza Virus H1N1 Infection in A549 Cells and its Immunomodulatory Mechanism, Beijing University of traditional Chinese Medicine, Beijing, China, 2017.

[76] X. T. Liu, L. G. Gu, and D. Y. Deng, "Effects of baicalin and luteolin-7-O-glucoside on NF- $\kappa$ B signaling pathway in A549 cells infected with influenza virus H1N1 in vitro," China Journal of Traditional Chinese Medicine and Pharmacy, vol. 31, no. 5, pp. 1937-1941, 2016.

[77] W. Zhang, J. J. Zhang, Q. F. Guo, L. Chen, and Z. H. Yi, "Advances in pharmacological studies of patchouli alcohol," Chinese Journal of Experimental Traditional Medical Formulae, vol. 26, no. 3, pp. 213-221, 2020.

[78] D. Yue, C. Z. Yu, C. Liang, D. Gang, W. Z. Zhong, and X. Wei, "Antiviral activity of chlorogenic acid against influenza A (H1N1/H3N2) virus and its inhibition of neuraminidase," Scientific Reports, vol. 7, 2017.

[79] M. G. E. Karar, M. F. Matei, R. Jaiswal, S. Illenberger, and N. Kuhnert, "Neuraminidase inhibition of dietary chlorogenic acids and derivatives-potential antivirals from dietary sources," Food \& Function, vol. 7, 2016.

[80] D. Li, P. Peng, and Z. Ke, "Forsythoside a controls influenza A virus infection and improves the prognosis by inhibiting virus replication in mice," Molecules, vol. 21, no. 5, 2016.

[81] F. A. Bellesi, V. M. P. Ruiz-Henestrosa, J. Maldonado-Valderrama, T. Del Castillo Santaella, and A. M. R. Pilosof, "Comparative interfacial in vitro digestion of protein and polysaccharide oil/water films," Colloids and Surfaces B: Biointerfaces, vol. 161, pp. 547-554, 2018.

[82] M. F. Zhang and Y. Q. Shen, "Glucocorticoid-like effect of glycyrrhizic acid and its aglycone glycyrrhetic acid," Drugs \& Clinic, vol. 26, no. 6, pp. 448-452, 2011.

[83] L. H. Su and J. G. Saeng, "Chrysophanol mitigates T cell activation by regulating the expression of CD40 ligand in activated T cells," International Journal of Molecular Sciences, vol. 21 , no. 17, 2020.

[84] R. F. Zhang, Z. F. Yang, and S. F. Jiang, "Research progress on pharmacological and toxicological effects of peppermint oil," China Pharmaceuticals, vol. 21, no. 19, pp. 1-3, 2012. 